\title{
Decision-Making in Ship Collision Avoidance based on Cat-Swarm Biological Algorithm
}

\author{
Zhaokun WEI ${ }^{1, a}$, Kang $\mathrm{ZHOU}^{2, \mathrm{~b}}$ and Ming WEI $\mathrm{W}^{3, \mathrm{c}}$ \\ ${ }^{1}$ Transportation Management College, Dalian Maritime University, Dalian, China \\ ${ }^{2}$ Navigation College, Dalian Maritime University, Dalian, China \\ ${ }^{3}$ Qingdao Harbour Vocational \& Technical College, Qingdao, China \\ aweizhaokun@dlmu.edu.cn, 'bhoukang@dlmu.edu.cn, 'wming858@163.com
}

Keywords: Decision-Making, Ship Collision Avoidance, Cat-Swarm Biological Algorithm

\begin{abstract}
Due to complicated maritime traffic condition, for the sake of offering collision alert and efficient collision avoidance action to mitigate navigators' working burden and fatigue and ensure navigation safety, this paper sets the fuel consumption as the objective function and combine International Regulations for Preventing Collisions at Seas(COLREGS) to provide the optimal collision avoidance action(including rudder steering timing, truing angle, reorientation timing and reorientation angle) through Cat-Swarm Biological Algorithm
\end{abstract}

\section{Introduction}

Above $80 \%$ of collision accidents are attributed to human error at sea according to historical statistics. As the shipping technology developing, the bridge equipment can offer more and more relevant information. Increasing information lowers the probability of accurate judgement for ship encounter situation timely[1]. More automatic navigating can reduce collision accidents which result from human error, as well as mitigate navigators' fatigue. The research on intelligent ship collision avoidance decision-making support have substantial meaning for shipping industry.

The problem of decision-making in ship collision avoidance has attracted attention of many researchers in the past. Ugur Simsir, Mehmat Fatih Amasyah et al[2] predicted the ship position in three minutes through analytical neural network based on ship maneuverability and ship encounter parameters. Through this, it can judge whether collision danger exists between two ships. If collision danger exists, predict the rudder steering timing. Lokukaluge P.Perera, Victor Ferrari[3] proposed a computer simulation to model the ship collision avoidance decision-making using a real ship model. Wentao Zheng, Yuanzhou Zheng[4] extract and acquire the basic ship information from the ship motion images, such as length, width, position and speed through Gauss Hybrid Model, in order to ensure the ship's motion situation and determine a collision danger level. Then it can make a collision alert and propose the collision avoidance decision-making. Guoquan Chen, Lina Li[5] focused on open sea and used artificial intelligent collision avoidance decision-making theory to simulate typical decision-making cases. Yueying Wang, Ashim Kumar Debnath[6] offer a collision avoidance model based on three typical collision avoidance action(speed altering, rudder steering, speed altering and rudder steering).

To solve the problem of ship collision avoidance, this study builds a nonlinear mathematical model Which considers crossing and head-on situation. Cat-swarm biological algorithm which is heuristic algorithm is adopted for solving the model.

The paper is organized as follows. Section 2 introduce ships encounter situation which include head-on, crossing, overtaking. Section 3 gives notation and decision parameters of target ship before building model. Section 4 develops mathematical model. Section 5 provides cat-swarm biological algorithm for solving proposed model. Section 5 carries out numerical experiments based on AIS data. Finally, a conclusion is given in section 6 . 


\section{Ship Encounter Situation and COLREGS}

The The ship encounter situation can mainly be divided into three types: head-on, overtaking and crossing.

(1) Head-on: when two ships are meeting on reciprocal ore nearly reciprocal courses so as to involve risk of collision and only when two ships see each other. In this situation no yielding relationships exists between the two ships. Regulations require each ship shall alter course to starboard so that each ship shall pass on the port of the other.

(2) Crossing: when two ships encounter each other in cross-over courses and pose danger, Regulations require a ship to yield to the other ship on the starboard ad avoid crossing the front of the other ship if conditions allow.

(3) Overtaking: an overtaking situation is defined, except when the two ships in sight of on another, as one ship is abaft the other ship abeam in an angle larger than 22.5 degree. At night, only sternlight is visible, not the sidelight and any ship overtaking any other shall keep out of the way of the ship being overtaken.

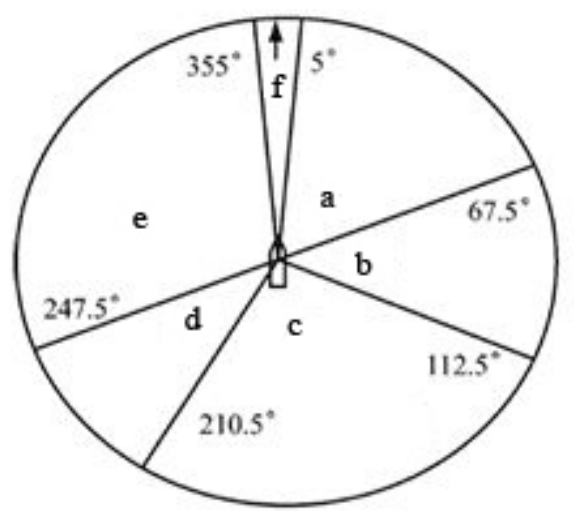

Fig.1 Ship encounter situation

In accordance with the OCLREGS, this study focuses on the encounter situation of two ships and divides action modes into six regions labeled $a$ to $f$, as shown in Fig.1. A head-on situation is created when ship encounters another ship in region $f\left(005^{\circ} \sim 355^{\circ}\right)$; Two ships encountering each other in region $a, b$ and $e$ are in crossing situation. If ship 1 is in an overtaking situation being passed by any ship from the $c\left(112.5^{\circ} \sim 210^{\circ}\right)$ or $d\left(210^{\circ} \sim 247.5^{\circ}\right)$ regions, it is usually required to keep course and speed. This paper focuses on ship collision avoidance decision-making research in head-on and crossing situation based on relevant regulations about overtaking situation[7].

\section{Decision Parameters of Target Ship}

The frequently-used decision parameters in decision-making model include the course of the own ship $c_{o}$, the course of the target ship $c_{t}$, the relative course of the two ships $c_{o t}$, the speed of the own ship $v_{o}$, the speed of the target ship $v_{t}$, the relative speed of the two ships $v_{r}$, the relative bearing of the target ship to the own ship $q$, the distance between the two ships $d, d_{c p a}$ (the distance between the own ship and the Closest Point of Approach) and $t_{c p a}$ (the time of the own ship reaching the Closest Point of Approach).

$$
\begin{aligned}
& c_{o t}=c_{o}-c_{t} \\
& v_{r}=\left(v_{t}^{2}+v_{o}^{2}-2 v_{t} v_{o} \cos c_{o t}\right)^{1 / 2}
\end{aligned}
$$




$$
\begin{aligned}
& \left\{\begin{array}{cc}
a=\arccos \left(v_{o}{ }^{2}+v_{r}{ }^{2}-v_{t}{ }^{2}\right) / 2 v_{o} v_{r} & c_{o t} \geq 0 \\
a=-\arccos \left(v_{o}{ }^{2}+v^{2}-v_{t}^{2}\right) / 2 v_{o} v_{r} & c_{o t}<0
\end{array}\right. \\
& \left\{\begin{array}{c}
d_{c p a}=d \sin \beta \\
t_{c p a}=d \cos \beta / v_{r} \\
\beta=a-q \quad c_{o t} \geq 0 \\
\beta=q-a \quad c_{o t} \leq 0
\end{array}\right.
\end{aligned}
$$

Equation 1 equals the interaction angle between own ship and the target ship before ships' turning, equation 2 provides the relative speed of the target ship according to own ship before the two ships' turning, equation 3 is the interaction angle between the direction of the relative motion of the target ship and own ship's heading; equation 4 is the $d_{c p a}$ and $t_{c p a}$ before ships' turning. The geometrical relationship is displayed in Fig.2.

$$
\begin{aligned}
& c_{o^{\prime} t}=c_{o^{\prime}}-c_{t} \\
& v_{r}^{\prime}=\left(v_{t}^{2}+v_{o}^{2}-2 v_{t} v_{o} \cos c_{o^{\prime} t}\right)^{1 / 2} \\
& \left\{\begin{array}{l}
b=\arccos \left(v_{o}^{2}+v_{r}^{\prime 2}-v_{t}^{2}\right) / 2 v_{o} v_{r}^{\prime} \quad c_{o^{\prime} t} \geq 0 \\
b=-\arccos \left(v_{o}^{2}+v_{r}^{\prime 2}-v_{t}^{2}\right) / 2 v_{o} v_{r}^{\prime} \quad c_{o^{\prime} t}<0
\end{array}\right. \\
& \left\{\begin{array}{c}
d_{c p a_{1}}=d \sin \theta \\
t_{c p a_{1}}=d \cos \theta / v_{r}^{\prime} \\
\theta=b+\Delta c-Q \quad c_{o^{\prime} t} \geq 0 \\
\theta=Q-b-\Delta c \quad c_{o^{\prime} t}<0
\end{array}\right.
\end{aligned}
$$

Equation 5 equals the interaction angle between own ship and the target ship after ships' turning, equation 6 provides the relative speed of the target ship according to own ship after the two ships' turning, equation 7 is the interaction angle between the direction of the relative motion of the target ship and own ship's heading after own ship' turning; equation 8 is the $d_{c p a}$ and $t_{c p a}$ after ships' turning. Their geometrical relationship is displayed is Fig.3.

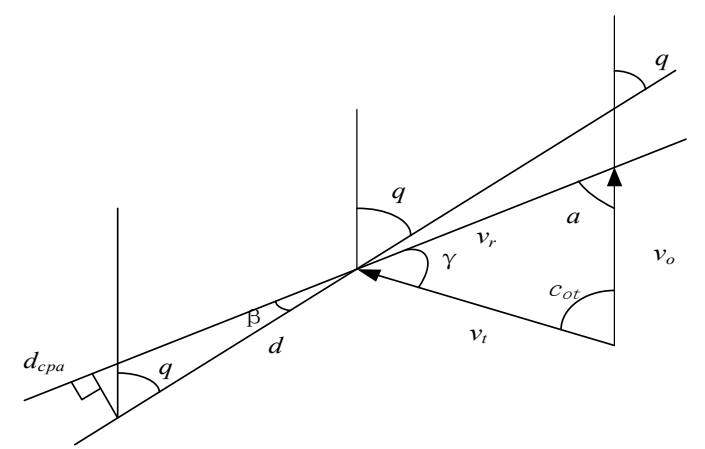

Fig.2. Geometrical situation before turning 


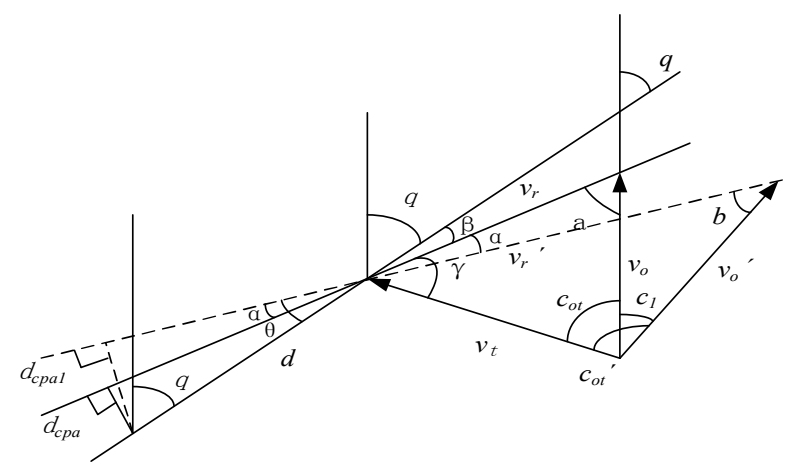

Fig.3. Geometrical situation after turning

\section{Mathematical Model}

\section{Problem Description}

COLREGS require that ships take relevant collision avoidance actions according to the regulation rules in the crossing and head-on situations. This paper establishes the optimization model to generate the basis for collision avoidance timing and turning angle, navigating timing after turning, timing of ease rudder and rudder angle of course again, pass each other in a safety distance to minimize the fuel consumption during collision avoidance. Offer the following assumptions to facilitate the establishment of the model.

(1) The ship's turning can be finished in an extremely short time after rudder steering, so ignore the turning time.

(2) Returning to the original route after collision avoidance procedure.

(3) No consideration of collision avoidance relating to other ships when own ship takes collision avoidance action.

(4) Without consideration of overtaking situation in accordance with COLREGS.

(5)No consideration of the two ships' collision avoidance actions with the aid of VHF communication.

Table1. Notation definition

\begin{tabular}{|c|c|}
\hline Notation & Definition or explanation \\
\hline$t_{b}$ & The navigating time before the ship taking turning action \\
\hline$t_{s}$ & The navigating time in the new course after turning \\
\hline$c_{1}$ & The ship's new heading course after turning \\
\hline$c_{2}$ & The course during reorientation \\
\hline$v$ & The ship's speed \\
\hline$g(v)$ & Fuel consumption per nautical mile \\
\hline$l$ & $\begin{array}{l}\text { The navigating distance during the whole collision avoidance } \\
\text { action }\end{array}$ \\
\hline$d_{s}$ & Preset safety distance for passing each other \\
\hline
\end{tabular}

\section{Mathematical Model}

$\min \lg (v)$

Subject to:

$30^{\circ} \leq c_{1} \leq 90^{\circ}$ 


$$
\begin{aligned}
& -60^{\circ} \leq c_{2} \leq-30^{\circ} \\
& t_{c p a} \leq t_{2} \leq 60 \\
& 0 \leq t_{1} \leq t_{c p a} \\
& d_{s} \geq d_{c p a} \\
& d_{s} \leq d_{c p a_{1}} \\
& t_{1} \geq 0, t_{2} \geq 0
\end{aligned}
$$

The objective function (9) minimizes the fuel consumption during collision avoidance action. Eq. (10) and (11) guarantees the requirement that avoiding small rudder steering angles according to COLREGS. Constraint (12) enforce the lasting time of collision avoidance action. Constraint (13) defines the ship's turning timing. Eq. (14) and (15) are introduced to guarantee the efficiency of collision avoidance action. Constraint (16) requires that $t_{1}$ and $t_{2}$ are non-negative.

\section{Solution Algorithm}

In recent years many researchers have proposed many artificial intelligent algorithms which are evolved through imitation of some biological animals' behaviors. Though all cats are, in most time, inactive and if you spend some time to observe the existence of cats and you may find that the cats spend most of the time on resting and peeping situation when they are awake, all cats have a strong curiosity and the alertness of cats are very high. They always stay alert even though they are resting. Thus, you can simply find that the cats usually look lazy, lying somewhere, but opening their eyes hugely looking around. Once discovering the targets, cats can capture the prey swiftly. The behaviors of cats can be divided into two sub-mode, i.e. tracing mode in which cats are looking around in lazy situation and seeking mode in which cats are tracing dynamic targets[8]. The solution procedure as follows:

Step 1. Initialize the cat swarm. Every cat has its own position composed of four dimensions: $t_{1}$, $t_{2}, \quad c_{1}, c_{2}, v$.

Step 2. Divide the cat swarm into groups, tracing mode and seeking mode, at random based on the grouping rate.

Step 3. Update the cats' positions via cats' mode identified by the mode flag. If in seeking mode, then activate the seeking mode; otherwise, behaviors of the tracing mode are activated. Resembling Particle Swarm Algorithm, in the every iteration in tracing mode, the cat will update its own position through tracing an extreme value which is the optimal solution in the current whole cat swarm. This tracing mode makes the cats approximate towards the optimal solution in the whole situation. Then update the cat's own position with this whole optimal position in accordance with Eq. (17) and Eq. (18).

$$
\begin{aligned}
& v_{k}{ }^{d}(t+1)=v_{k}{ }^{d}(t)+c \times \text { rand } \times\left(x_{\text {best }}{ }^{d}(t)-x_{k}{ }^{d}(t)\right) \\
& x_{k}{ }^{d}(t+1)=x_{k}{ }^{d}(t)+v_{k}{ }^{d}(t+1)
\end{aligned}
$$

$\mathrm{k}$ denotes the $\mathrm{k}^{\text {th }}$ cat, $\mathrm{d}$ denotes the $\mathrm{d}^{\text {th }}$ dimension of the $\mathrm{k}^{\text {th }}$ cat. In seeking mode, cats replicate their own positions and put these duplicates of positions into memory pool. Then change the duplicate in 
the memory pool via mutation operator to let all the duplicates acquire new positions. Replace the current position with the one which have the highest fitness level.

Step 4. Calculate the fitness value of each cat via the fitness function. Record and keep the cat with the optimal fitness value.

Step 5. Check whether it satisfy the terminate condition. Output the optimal solution and end the procedure if positive. Otherwise, repeat step 2.

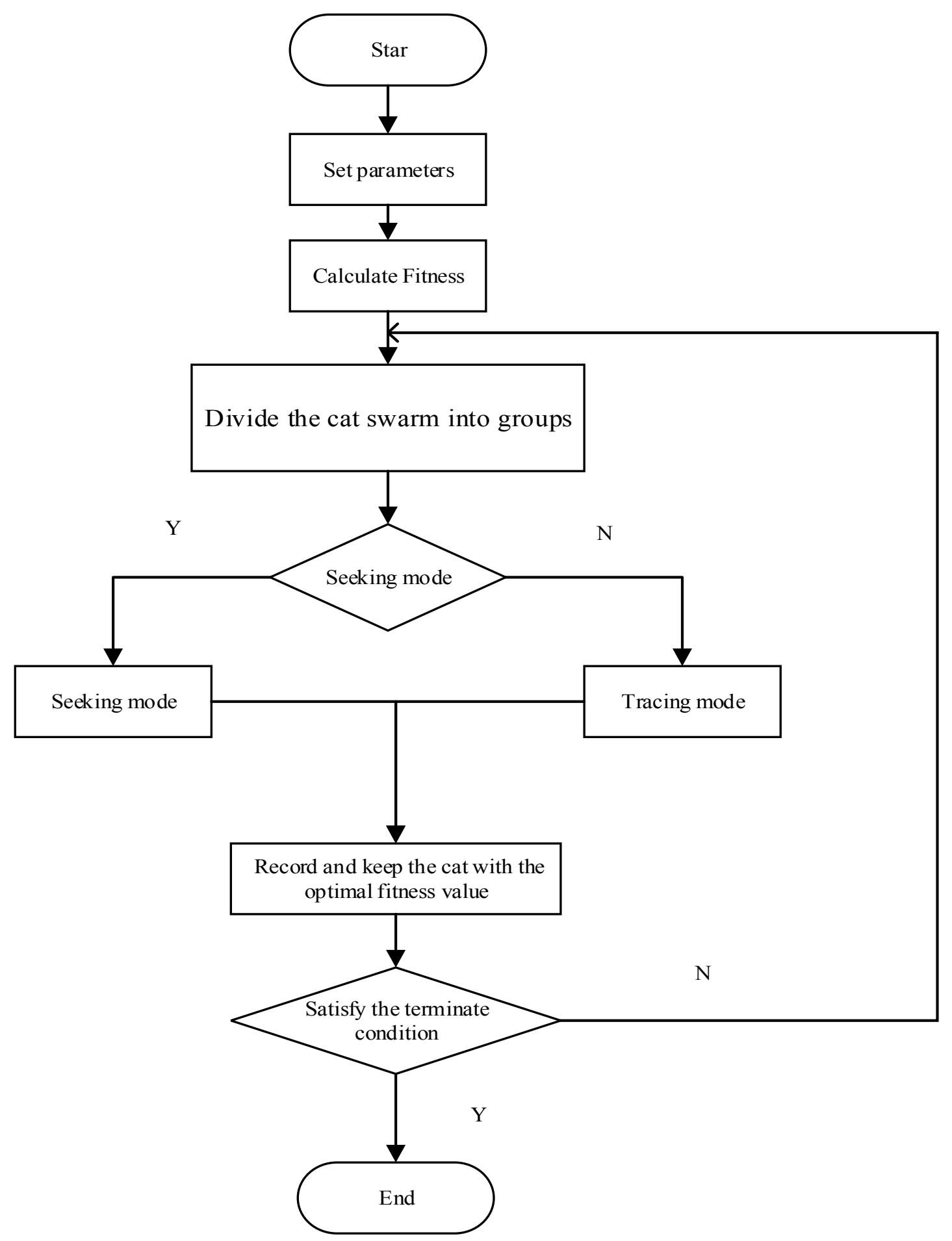

Fig.4. Process diagram of Cat Swarm Algorithm 
The data resource of the emulation case is the dynamic AIS information. Generate the head-on and crossing situations in this emulation. The course of own ship is $000^{\circ}$. The target ship with the speed of 12 knots is $30^{\circ}$ to the starboard of own ship and 20 nautical miles away from own ship. Assume that the two ships just turn around when in collision avoidance action. The optimization results are displayed in Table 2.

Tab. 2 Optimization Result 1

\begin{tabular}{|c|l|l|l|l|l|}
\hline Target Ship & $t_{1}$ & $t_{2}$ & $c_{1}$ & $c_{2}$ & $v$ \\
\hline $250^{\circ}$ & $2.85 \mathrm{~min}$ & $37.61 \mathrm{~min}$ & $30^{\circ}$ & $48^{\circ}$ & $8 \mathrm{kn}$ \\
\hline $181^{\circ}$ & $10.8 \mathrm{~min}$ & $9.7 \mathrm{~min}$ & $32^{\circ}$ & $38^{\circ}$ & $10 \mathrm{kn}$ \\
\hline $200^{\circ}$ & $3 \mathrm{~min}$ & $31 \mathrm{~min}$ & $30^{\circ}$ & $53^{\circ}$ & $8.5 \mathrm{kn}$ \\
\hline
\end{tabular}

Tab. 3 Optimization Results 2

\begin{tabular}{|l|l|l|l|l|l|}
\hline Target Ship & $t_{1}$ & $t_{2}$ & $c_{1}$ & $c_{2}$ & $v$ \\
\hline $250^{\circ}$ & $17.82 \mathrm{~min}$ & $19.34 \mathrm{~min}$ & $32^{\circ}$ & $45^{\circ}$ & $8.6 \mathrm{kn}$ \\
\hline $181^{\circ}$ & $4.6 \mathrm{~min}$ & $13.63 \mathrm{~min}$ & $45^{\circ}$ & $57^{\circ}$ & $11.2 \mathrm{kn}$ \\
\hline $200^{\circ}$ & $4.73 \mathrm{~min}$ & $16.17 \mathrm{~min}$ & $65^{\circ}$ & $58^{\circ}$ & $8.6 \mathrm{kn}$ \\
\hline
\end{tabular}

Through the comparison of Tab2 and Tab3, we can get that with consideration of fuel consumption, own ship took collision avoidance action earlier than it does which set the shortest collision avoidance route as the objective function. The collision avoidance action considering the shortest collision avoidance route can guarantee the avoidance route shortest. By contrast, the decision-making based on fuel consumption is for the sake of economics. So the final output of this optimization model is to steer own ship earlier and then slow the speed down, which can guarantee the minimum fuel consumption and as well as the safe pass in safety distance, however can not guarantee the shortest route.

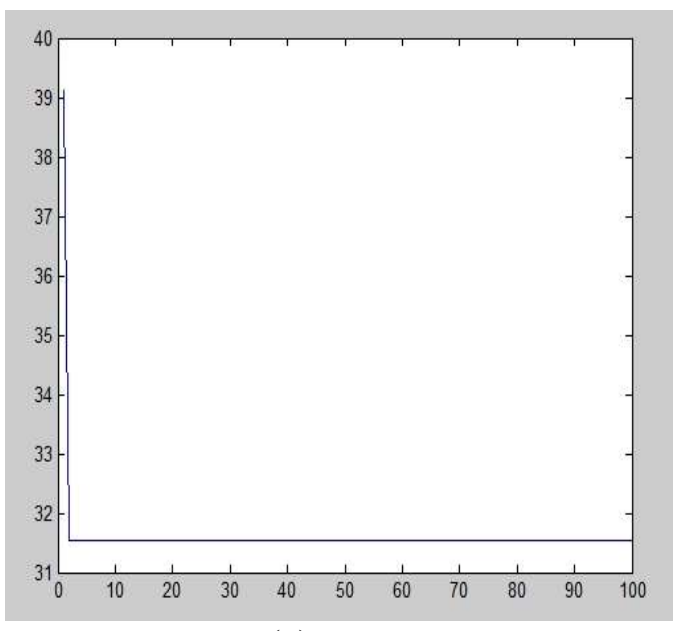

(a)

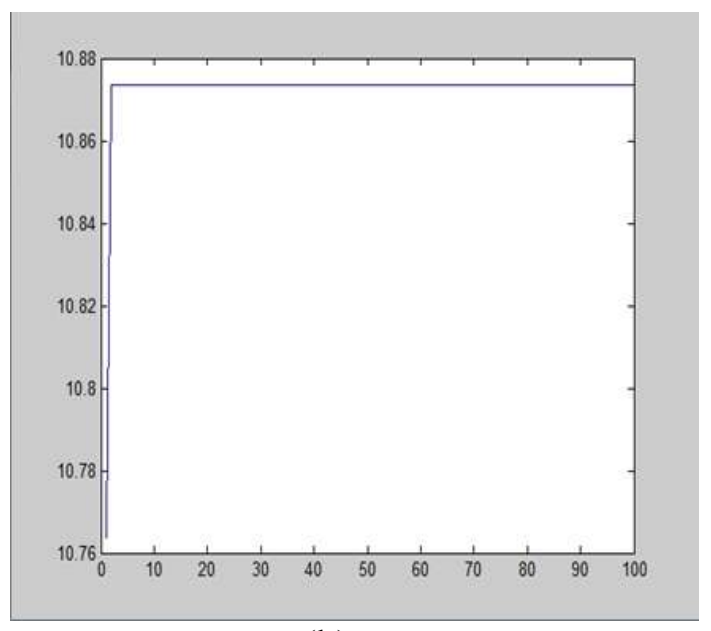

(b)

Fig.4. Iteration change of $t_{1}$ and $t_{2}$ 


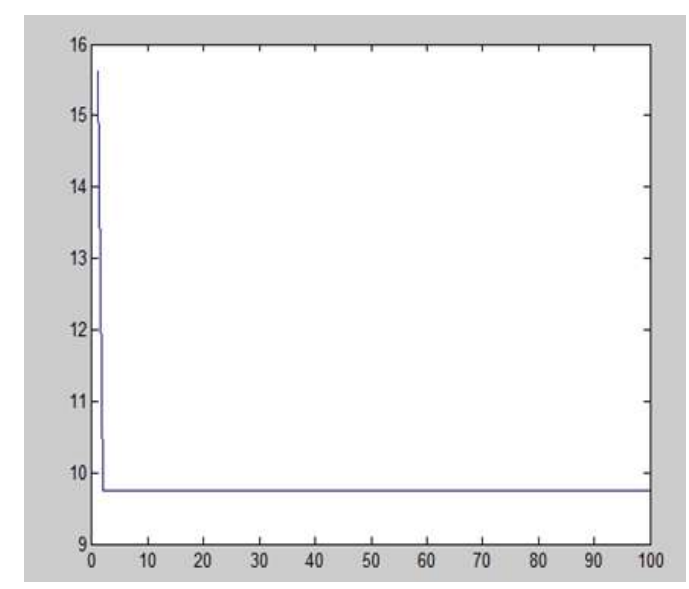

(a)

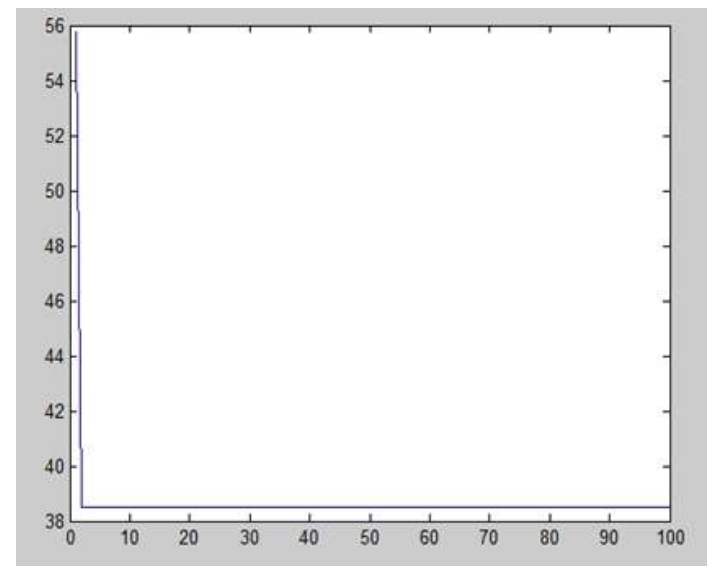

(b)

Fig.5. Iteration change of $c_{1}$ and $c_{2}$

The horizontal axes of Fig.4 and Fig.5 are iteration times. The vertical axes of them are optimization results of $t_{1} 、 t_{2} 、 c_{1}$ and $c_{2}$. From the above, we can get the optimal output in ten times of iteration via cat swarm algorithm which has higher iteration efficiency.

\section{Conclusion}

Academic research always regards the ship collision avoidance safely as a hot topic. And most of collision avoidance models are established in the view of the shortest route. This paper establish a mathematic model of ship collision avoidance based on fuel consumption with consideration of economics, which is aimed to get the minimum fuel consumption during ship collision avoidance, as well as the two ships passing each other in safety distance. Considering the mixed integer nonlinear programming, we apply the cat swarm algorithm to solve this optimization model and verify the optimal solution via numerical emulation; finally approve the model to be efficient and the algorithm feasible. And this optimization model can provide technique support for ship collision avoidance.

\section{Acknowledgement}

The authors are grateful to the professor Xinlian Xie who gives guidance during the research and other colleagues who help collect data and materials

\section{References}

[1] Bai Yiming, Han Xinjie, Meng Xianyao. Optimization of Strategy for Ship Collision Avoidance with Danger Model Immune Algorithm. NAVIGATION OF CHIAN. 35(2012) 29-32.

[2] Ugur Simsir, Mehmet Fatih Amasyah. Decision support system for collision avoidance of vessels.Applied Soft Computing, 2014, pp:369-378

[3] Lokukaluge P. Perera, Victor Ferrari. Experimental Evaluations on Ship Autonomous Navigation and Collision Avoidance by Intelligent Guidance. Oceanic Engineering. 40(2015) 374-386.

[4] Wentao Zhang, Yuanzhou Zheng. Intelligent Ship-Bridge Collision Avoidance Algorithm Research Based on A Modified Gaussian Mixture Model. Multimedia Technology, 2011, pp:6414-6419

[5] Guoquan Chen, Lina Li. Mechanism and Simulation of Personifying Intelligent Decision-making for Vessel Collision Avoidance. Computer Application and System Modeling. 2010, pp:681-686 
[6] Yue Ying Wang, Ashim Kumar Debnath. MODELING COLLISION AVOIDANCE DECISIONS IN NAVGATION.In Proceedings of 10th Asian Conference on Marine Simulation and Simulator Research. TaiWan, 2010.

[7] LIU Dexin, WU Zhaolin, JIA Chuanying. Decision-making model of Dcpa,Tcpa and object's movement parameter. Journal of Dalian Maritime University, 30(2004) 22-25.

[8] S.C.Chu, and P.W.Tsai. Computational Intelligence Based on behavior of Cat. International Journal of Innovative Computing, Information and Control, 3(2007) 163-173. 